\title{
Familiarity impacts person perception
}

\author{
TERESA GARCIA-MAROUES ${ }^{1 *}$ \\ AND DIANE M. MACKIE ${ }^{2}$ \\ ${ }^{1}$ Instituto Superior de Psicologia Aplicada, \\ Lisbon, Portugal \\ ${ }^{2}$ University of California, Santa Barbara, \\ California, USA
}

\begin{abstract}
We investigated the effects of familiarity on person perception. We predicted that familiarity would increase non-analytic processing, reducing attention to and the impact of individuating information, and increasing the impact of category labels on judgments about a target person. In two studies participants read either incriminating or exculpatory individuating information about a defendant in a criminal case and made judgments of guilt. In Study 1, participants were subliminally exposed to the defendant's photo, another matched photo, or no photo before seeing the evidence. Participants familiar with the defendant's photo both processed and used the individuating information less. In Study 2, participants were subtly made familiar or not with the incriminating and exculpatory information itself, and the defendant was described either as a priest or as a skinhead. Familiarity with the information reduced attention to its content and also tended to increase reliance on category information in guilt judgments. Copyright (C) 2006 John Wiley \& Sons, Ltd.
\end{abstract}

Imagine that as you browse the morning paper, an article on a local court case catches your eye. A brief article summarizing the arguments made by the prosecution and defense is accompanied by a small photo of the accused. Having never before seen the face before you go ahead and read the information, drawing your own conclusions. Now imagine instead that as you turned to read the facts of the case the face looked vaguely familiar. How might that vague glimmer of familiarity affect any judgment you make about the individual?

We have argued that the experience (whether conscious or not) of such familiarity regulates information processing, making analytic processing less likely (Garcia-Marques \& Mackie, 2000, 2001). We thus take a dual process view that human information processing involves two distinct modes of computation (see Abelson, 1994; Sloman, 1996; Smith \& DeCoster, 2000, for reviews): nonanalytic processing (characterized by the mere activation of a way to deal with a focal stimulus), and

*Correspondence to: Teresa Garcia-Marques, Instituto Superior de Psicologia Aplicada, Rua Jardim do Tabaco, 34, 1149-041 Lisbon, Portugal. E-mail: gmarques@ispa.pt 
analytic processing (in which the particulars of a situation are carefully and systematically analyzed). When stimulus situations match memory representations, initial bottom-up processing occurs with an "ease" or "fluency" that results in an (implicit) feeling of "similarity," "recognition," or "familiarity" (Eich, 1982; Fiske, 1982; Gillund \& Shiffrin, 1984; Hintzman, 1988; Humphreys, Bain, \& Pike, 1989; Jacoby \& Dallas, 1981; Murdock, 1982). This familiarity in turn depresses analytic processing, saving limited analytic processing resources for novel situations rather than situations that have been dealt with before (Johnston \& Hawley, 1994).

Research findings in several areas are consistent with these arguments. Research on problem solving, for example, has demonstrated that a "feeling of knowing" regulates the process underlying problem solving (Reder \& Ritter, 1992; Schunn, Reder, Nhouyvanisvong, Richards, \& Stroffolino, 1997). Individuals' quick judgments about whether they felt they could retrieve an answer to a problem or whether they had to compute it were independent of actually knowing the answer but closely dependent on the familiarity of the situation. Familiar situations gave participants a "feeling" that they "knew" the answer, and thus promoted less effortful, top-down, retrieval strategies. Unfamiliar situations, in contrast, triggered more effortful bottom-up computational strategies. Thus, a "feeling of knowing" caused by familiarity acted as a critical signal to switch the cognitive system between nonanalytic and analytic processing modes.

We have also shown that prior exposure to a persuasive appeal decreases the likelihood of its elaboration (Claypool, Mackie, Garcia-Marques, McIntosh, \& Udall, 2004; Garcia-Marques \& Mackie, 2001). Using a typical dual processing paradigm, we were able to show that novel arguments were systematically processed as evidenced by differential agreement with strong and weak arguments. In contrast, however, people responded to previously encountered messages with less elaboration, eliminating the differential acceptance of strong and weak messages.

Of most relevance, we (Smith, Miller, Maitner, Crump, Garcia-Marques, \& Mackie, 2006) demonstrated in two experiments that repeated supraliminal exposure to a target person increased the occupational stereotyping of that target. In these experiments, participants were repeatedly exposed to the photos of some targets about whom they subsequently learned an occupational label and some mildly counter-stereotypic information. Participants later rated the targets on several traits, some of which were related to the occupational stereotype. Participants made more stereotypic ratings of targets to whom they had previously been exposed. In the second experiment, drawing attention to the possibility of multiple exposure undermined this effect, implicating the role of familiarity. The results were thus consistent with the idea that familiarity functioned as a regulator of processing mode, such that information about familiar objects received less analytic processing.

All these approaches suggest that familiarity with a situation triggers non-analytic rather than analytic processing. In this research we extend our claim to the impression formation or person perception literature adding new insights about how familiarity might be elicited. According to dual process models of person impression, judgments of a target can be based either on analytic individuation, the piecemeal processing and combination of individual pieces of information about the target, or on non-analytic categorical processing, the drawing of inferences about target characteristics based on category membership (Brewer, 1988; Fiske \& Neuburg, 1990; Neuberg \& Fiske, 1987). Individuation is more effortful than categorybased processing, as evidenced by the fact that participants who engage in piecemeal processing of a target's attributes spend more time looking at and take longer to read and rate the information (Fiske, Neuberg, Pratto, \& Allman, 1986, cited in Fiske \& Neuburg, 1990; Fiske, Neuberg, Beattie, \& Milberg, 1987; Neuberg \& Fiske, 1987) than do other participants. Also consistent with this idea is the finding that individuation is undermined by cognitive capacity constraints (Bodenhausen, 1990). 
If familiarity regulates whether information is processed analytically or non-analytically, we expect familiarity to influence the differential impact of individuating information on subsequent person impression judgments. A similar assumption is implicit in Fiske's claim that category-based judgments depend on the degree of similarity between the target and previously stored structures (Fiske \& Pavelchak, 1986; Neuberg \& Fiske, 1987). Category-based processing has priority over individuation, and only when target features fail to match typical category features does more attribute-based processing take place. In our terms, the match of perceived features to stored categories entails familiarity, and a consequent decrease of detailed resource intensive processing. Thus, our claim goes further than Fiske's view, since we assume that familiarity can be triggered by many different features of the context (and not just the match between target features and category representations), but will nevertheless have the same impact on processing.

We present two studies of impression formation that manipulate prior exposure to different aspects of the processing situation to produce familiarity. If familiarity triggers non-analytic processing, we expected judgments made under conditions of familiarity to show less impact of individuating information presented about the target than judgments made under novel conditions, regardless of how familiarity was induced.

\section{STUDY 1}

In the first study prior subliminal exposure to the photo of a crime suspect was used to induce a sense of familiarity during later presentation of individuating information relevant to the accused's guilt or innocence. We had two main hypotheses. First, we expected an unsurprising overall impact of individuating information, with incriminating evidence producing stronger judgments of guilt than exculpatory evidence. Second, and more critical theoretically, we expected that compared to conditions in which familiarity was not induced, the familiarity induced by prior exposure to the target would reduce or eliminate the impact of individuating information on judgments of guilt. We also wished to eliminate an alternative explanation that might be offered for such findings. Social judgeability (Yzerbyt, Dardenne, \& Leyens, 1998) suggests that when people feel they have some prior information about a topic, they feel confident that they have sufficient information on which to make a judgment and thus fail to process additional relevant information analytically. We measured participants' perceptions of having sufficient information on which to base a judgment so that we could analytically assess the role of judgeability in our results.

\section{Method}

\section{Participants and Design}

Participants were 144 undergraduates (72\% females) at the Instituto Superior de Psicologia Aplicada (ISPA) in Lisbon, Portugal (mean age $=20$ years old) who volunteered to complete this study. They were randomly distributed to the 12 cells of a between-subjects factorial design 3 (no prior exposure vs. prior exposure vs. irrelevant prior exposure) $\times 2$ (incriminating vs. exculpatory individuating evidence) $\times 2$ (stimulus replication of target face). Participants completed the experiment in groups of 10-20, with random assignment to conditions within each session. 


\section{Procedure}

Participants were seated at computers and asked to complete a number of different studies. The first study was presented as a visual perception study and served to manipulate prior exposure to the target face. Two male target faces (A and B) were selected for use because they received neutral evaluations (on 7-point scales) of warmth $\left(M_{\mathrm{A}}=3.87 ; M_{\mathrm{B}}=4.12 ; t<1\right)$ and intelligence $\left(M_{\mathrm{A}}=3.87 ; M_{\mathrm{B}}=4.00 ; t<1\right)$ from 24 other members of the subject population not involved in the study. Both targets were also perceived as equally non-aggressive $\left(M_{\mathrm{A}}=2.42 ; M_{\mathrm{B}}=2.83 ; t(23)=1.59\right.$, $p<.125)$.

Manipulation of prior exposure Participants were focused on the center of the screen (where $\mathrm{a}+$ was presented) and then pressed the space bar to activate a sequence of five different pictures (e.g., a tree, a car, a house, a rabbit, a cat) each presented for variable times ranging from 30 to 70 milliseconds. Between the third and fourth pictures participants were exposed for 18 milliseconds to a blank screen, the target photo, or a matched photo. To replicate across stimulus materials, each photo (A or B) was used as both target and matched control across conditions.

Participants were then asked to estimate how many objects had been presented; how many colored pictures were presented; and how many living things were presented. In addition they were asked to name the picture presented for the briefest time and the picture presented for the longest time. These questions allowed us to assess whether participants were conscious of the subliminal presentation of the face or not (if they were, the answer to the frequency estimate about living things would increase from three to four, for example). Three sequences of five objects were presented so that participants were exposed to three subliminal repetitions of the target.

Participants then completed a "second study," in which they identified the name of 10 European countries as quickly as possible (a filler task).

Person impression task Participants were told that the "third" study assessed people's abilities to serve as a member of a jury hearing a criminal case. The instructions stressed the need for concentration and that all the information about the case should be read carefully.

Following Bodenhausen and Lichtenstein (1987), we told participants they would be receiving information about a case ("Criminal Proceedings $N^{\circ}$ 190-23271") described as an assault occurring at a certain time and place committed by an unidentified individual who ran away leaving the victim unconscious.

Participants then viewed a photo of the alleged perpetrator (half the participants saw Photo A and half saw Photo B) accompanied by a description of the suspect as a 25-year-old single male resident of Lisbon, who denied the charges and was free on bail.

Presentation of exculpatory or incriminating individuating information Exculpatory or incriminating individuating information was then presented on the following screen as a brief summary of the investigation. Seven pieces of information about the crime were presented. In the exculpatory condition, participants saw four exculpatory, two incriminating, and one neutral item (in the following order: eiieeen). In the incriminating condition, four incriminating, two exculpatory, and one neutral item were presented (in the following order: ieeiiin). An example of an exculpatory item (in translation) was "No physical evidence either at the crime scene or on the suspect link him to the crime." An incriminating item was "The suspect was seen leaving a coffee shop near the scene of the 
crime about 10 minutes before the crime was committed." ${ }^{1}$ Participants took "all the time they felt necessary" to read this information.

\section{Dependent Variables}

Participants were then required to made several judgments, all on 9-point scales. Four scales comprised a measure of the suspect's perceived guilt: the defendant's guilt ("not at all likely to be the one who did it" to "extremely likely to be the one who did it"), a proposed verdict ("Sure he's not-guilty" to "Sure he's guilty), a recommended severity of sentence ("No time in jail" to "Several years in jail"), and a recommendation about whether the defendant should be detained in jail prior to trial ("I am sure that he does not need to be detained"; "I am sure that it is better to detain him").

Three other 9-point scales assessed the sufficiency of the information presented for making a decision (with anchors "insufficient for any decision" to "highly sufficient for a decision"); how incriminating the presented evidence was (anchored by "not at all" to "highly incriminating"); and how aggressive the suspect seemed to be (with scale anchors "not at all" to "highly aggressive").

\section{Results and Discussion}

Eleven participants were excluded from analysis because of indications that they had seen a human face during the exposure phase (seven reported having seen more than three living things and four reported having seen a "mask," "face," or "man"). Given that the subliminality of the stimulus repetition was essential to eliminating alternate explanations for any possible effects, it was important to provide the most conservative test of our hypotheses by excluding any participants who may have become aware of the stimulus repetition.

\section{Judgments of Guilt}

Responses to the four items used to assess guilt (Cronbach alpha $=.70$ ) were averaged, with high numbers reflecting higher guilt. Given that our hypotheses focused on specific comparisons of particular experimental conditions, we conducted planned contrasts using the overall error term $(\mathrm{Mse}=1.72)$. First, and as expected, participants based their judgments on evidence furnished about the case, $t(128)=4.32, p<.001$. A preponderance of incriminating evidence produced higher judgments of guilt $(M=5.42)$ than a preponderance of exculpatory information $\left(M=4.42, \eta^{2}=0.13\right)$. This difference also indicated that the manipulation of information was effective as intended.

Our main hypothesis was that the effect of individuating information would be reduced or eliminated by prior exposure to the target photo. To test this hypothesis two different planned contrasts were performed (Judd, Park, Yzerbyt, Gordijn, \& Muller, 2005). First, the differential impact of individuating information on guilt judgments following prior exposure to the target photo was contrasted with the differential impact of individuating information in the other two (no exposure,

\footnotetext{
${ }^{1}$ The incriminating and exculpatory items used were chosen from a larger pool on the basis of the ratings of 20 pilot participants, who evaluated how favorable or unfavorable each individual item was for the defendant using an 11-point scale anchored with -5 (extremely unfavorable) to +5 (extremely favorable). Twenty additional pre-test participants read the exculpatory version of the evidence and another 20 read the incriminating version. Both versions were rated on an 11-point scale ranging from 0 (not at all incriminating) to 10 (extremely incriminating). Results showed that the incriminating version was in fact seen as more incriminating $(M=5.80)$ than the exculpatory one $(M=4.45), t(38)=2.77, p<.01$.
} 
irrelevant exposure) conditions. As predicted, this interaction was significant, $t(128)=2.18, p<.03$, $\eta^{2}=0.03$. Consistent with our hypothesis, participants' guilt judgments were not differentially affected by individuating information following prior exposure to the relevant target (Minc $=4.90$, Mexc $=4.62 ; t<1$ ), but were strongly influenced by the individuating information in the other two conditions $(\mathrm{Minc}=5.58, \mathrm{Mexc}=4.32 ; t(128)=4.94, p<.001)$. Second, we contrasted the impact of individuating information on guilt judgments in the no exposure and irrelevant exposure conditions. As expected, the impact of the individuating information was present both when prior exposure was irrelevant $\left(\right.$ Minc $=5.75$, Mexc $\left.=4.09 ; t(128)=4.24, p<.001, \eta^{2}=0.12\right)$ and when there was no prior exposure $\left(\mathrm{Minc}=5.62, \mathrm{Mexc}=4.56 ; t(128)=2.76, p<.006, \eta^{2}=0.06\right.$; these conditions did not differ, $t(128)=1.28, p<.281)$. Consistent with our expectations, both these contrasts support that conclusion that familiarity with the target person, as operationalized by prior subliminal exposure to his photo, decreased the impact of individuating evidence on judgments (Figure 1).

The lack of significance associated with an overall effect of prior exposure $(t<1)$ on guilt judgments indicated that familiarity did not induce more positive judgments, as might be expected by the mere exposure hypothesis. Consistent with mere exposure, participants in the prior exposure condition rated the target with incriminating evidence more positively $(M=4.89)$ than in both other conditions $\left(M=5.68, t(128)=2.05, p<.043, \eta^{2}=0.03\right)$. However, not only is this effect not present with regard to exculpatory evidence $(t<1)$, but participants previously exposed to the target gave him more negative ratings $(M=4.63)$ than participants in both other conditions $(M=4.32)$, a result completely at odds with the operation of mere exposure.

We performed several analyses to see if the effect of prior exposure was mediated by increased perceptions of information sufficiency (a hypothesis derived from social judgeability theory). ANOVA of participants' sufficiency judgments revealed an impact of individuating information on perceived sufficiency, such that incriminating information was perceived to be more sufficient for a decision $(M=4.75)$ than was exculpatory information $\left(M=3.52, F(1,128)=13.55, p<.001, \eta^{2}=0.10\right)$; this effect is unsurprising given the negativity effect (Anderson, 1965; Dreben, Fiske, \& Hastie, 1979; Fiske, 1980). This effect was, however, qualified by prior exposure, $F(2,128)=2.83, p<.063$, $\eta^{2}=0.03$, indicating that the nature of the information made a difference to perceived sufficiency in both the no exposure and irrelevant exposure conditions $(\mathrm{Minc}=5.10, \mathrm{Mexc}=3.93$; $\mathrm{Minc}=5.22$, Mexc $=3.44$, respectively; overall $\left.t(128)=4.50, p<.001, \eta^{2}=0.014\right)$ but not in the prior exposure condition $($ Minc $=3.84$, Mexc $=3.32 ; t<1)$.

The fact that perception of information sufficiency, and thus perceived judgeability, was not affected by prior exposure itself $(F<1$; if anything prior exposure reduced rather than increased information sufficiency) was important in excluding a judgeabilty explanation of our results.

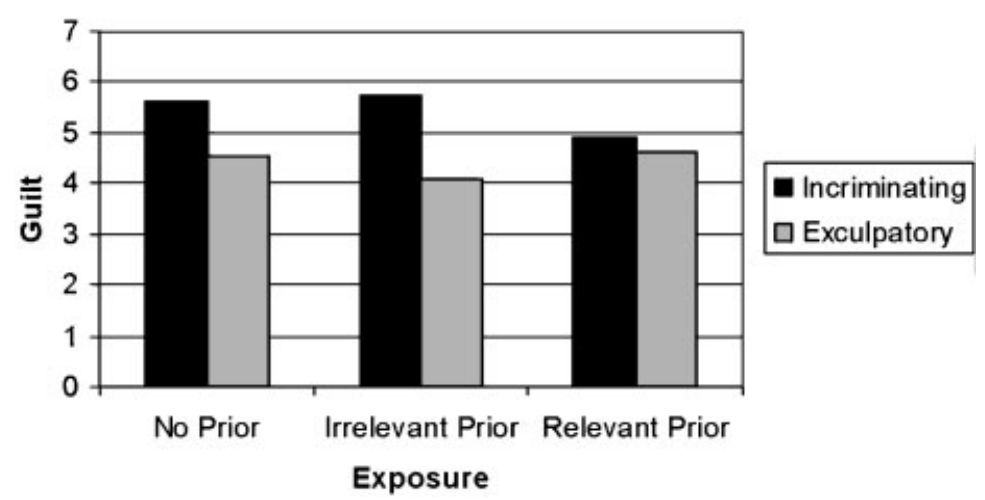

Figure 1. Guilt judgments as a function of prior exposure and nature of individuating information, Study 1 
However, since prior exposure made the perceived sufficiency of incriminatory and exculpatory information equal, it is possible that this effect contributed to the effect observed for judgments of guilt (perceived sufficiency and perceived guilt were significantly correlated, $r=0.57, t(133)=7.90$, $p<.001$. To test this hypothesis, the relevant planned contrasts were re-computed with sufficiency as a covariate. Controlling for sufficiency did not change the pattern of data previously observed. The significant interaction, $t(127)=1.99, p<.48, \eta^{2}=0.03$, suggested that the differential impact of individuating information present in the no exposure and irrelevant exposure conditions was eliminated when participants has been exposed to the target face before. Responses in the no exposure and irrelevant exposure conditions did not differ, $t(127)=1.42, p<.158$. These results show that although prior relevant exposure impacts the perceived sufficiency of incriminatory and exculpatory information, this impact does not explain our results. On the contrary, both effects are possible consequences of prior exposure reducing sensitivity to individuating information.

If participants in the prior exposure condition were indeed less sensitive to individuating information, we also expected those participants to be less sensitive to the incriminatory or exculpatory nature of that information. To test this idea, we contrasted ratings of how incriminating the information was in the relevant prior exposure condition compared to both other conditions combined, $\left(t(128)=3.16, p<.001, \eta^{2}=0.07\right)$. Post hoc comparisons showed that participants in both the no exposure and irrelevant exposure conditions combined were sensitive to the nature of the individuating information $\left(\right.$ Minc $\left.=5.13, \operatorname{Mexc}=3.62 ; t(128)=5.13, p<.001, \eta^{2}=0.17\right)$, whereas those in the relevant prior exposure condition were not $(\operatorname{Minc}=3.96$, Mexc $=3.60 ; t<1)$. Ratings in the irrelevant $($ Minc $=5.58$, Mexc $=3.60)$ and no exposure $($ Minc $=5.60$, Mexc $=3.32)$ conditions did not differ $(t<1)$.

Given that prior exposure had a similar impact on judgments both of how incriminating the information was and of guilt, it is not surprising that these two judgments were strongly related, $r=0.73, t(133)=12.26, p<.001$, suggesting that, as expected, the effect of prior exposure on perceived guilt was associated with reduced sensitivity to individuated information. To test this idea, we re-computed the two relevant planned contrasts (the qualification of the impact of the nature of information by prior exposure, and the unqualified effect of individuating information across the irrelevant and no exposure conditions) using judgments of how incriminating the information was as the covariate, expecting null effects. Results supported our assumptions, since the inclusion of this covariate eliminated the interaction of individuating information with prior exposure making all three conditions similar (all $t \mathrm{~s}<1$ ).

The results of this study indicated that prior exposure to a target's photo reduced the impact of individuating information on judgments about the target so that in fact perceivers were insensitive to its implications. These findings are consistent with our expectation that prior exposure reduces analytic processing. These results extend previous findings regarding the role of familiarity in impression formation in several regards. First, this is the first time that a reduction in processing has been demonstrated after familiarity has been induced via subliminal exposure. In our previous work, repetition of the stimulus was always supraliminal, thus opening the consequent reduction in processing to other possible interpretations such as boredom (in the case of reduced processing of repeated persuasive messages) or sufficiency (in the case of repeated judgments of people). In this case, participants appeared completely unaware of their prior exposure, whose effects were nevertheless predictably clear. Second, participants' perceptions of the presented evidence provide the first direct evidence of processing decrements following prior exposure in this paradigm. Our findings indicate that judgments made by participants in the prior exposure condition are less influenced by the nature of individuating information. This indirect indication of reduced processing thus extends to the person impression domain our earlier findings that under conditions of familiarity, attitude judgments are less influenced by strong and weak arguments (Claypool et al., 2004; Garcia-Marques \& Mackie, 2001) and 
stereotype judgments are less influenced by counter-stereotypic information (Smith et al., 2006) in this experiment, we were also able to show more directly that participants in the prior exposure condition were not as able to accurately judge the exculpatory and incriminating nature of highly relevant individuating information, consistent with our claim that familiarity reduces analytic processing.

Third, we produced familiarity's expected reduction of analytic processing even under conditions that neither restricted participants' capacity nor undermined their motivation. If anything, the context of and instructions given in the experimental setting might be expected to increase participants' motivation to careful process individuating information. Recall that participants in this experiment were told that the study assessed their ability to serve as a juror in a criminal trial, were asked to make judgments about someone's guilt or innocence, and were directly instructed to process all information carefully. These aspects of the experimental context are unique among studies of the impact of prior exposure reducing processing (Garcia-Marques, Mackie, Claypool, \& Garcia-Marques, 2004; Smith et al., 2006). In fact, experimentally induced high motivation reduced the impact of familiarity on the processing of persuasive information, although in the context of supraliminal repetition (Claypool et al., 2004). In the current study prior exposure nevertheless reduced analytic processing when participants were unaware of the exposure. Thus, these findings suggest that familiarity can reduce processing even in conditions of relatively high motivation to process.

Finally, we were also able to provide evidence inconsistent with a judgeability explanation of our effects. If social judgeability were at work, prior exposure should lead participants to feel more "entitled" to make a judgment. Our participants report exactly the opposite, and so of course there is no evidence that judgeability mediated the reduction in impact of information on guilt judgments in the prior exposure conditions.

There are two issues that need to be addressed to strengthen the claims that we are making here about prior exposure and reduced processing. The first issue has to do with the nature of our claim about prior exposure and familiarity. We are arguing that prior exposure to the target face reduces the likelihood of attending to individuated information about that person because re-exposure generates familiarity. We are thus arguing for a general effect of familiarity, not one that is specifically generated by this particular method of operationalizing familiarity. It is not that a target with a "familiar face" is perceived differently or that a familiar face induces different processing demands (e.g., Bruce \& Young's, 1986) but that perceiving (consciously or unconsciously) a familiar stimulus impacts how information is going to be processed. In our persuasion studies (Garcia-Marques \& Mackie, 2001) we made participants familiar not with the attitude object or the message source, but with the persuasive arguments themselves. We argued that it was the familiarity associated with being exposed to them again that engaged non-analytic processing. Generalizing this reasoning to the current context, we expected that making participants familiar with the criminal evidence itself would reduce analytic processing of its content, even though it also makes salient that very content. This constitutes a strong test of our hypothesis, since priming considerations might lead to the very opposite prediction. This also helps address the second issue, which is to rule out the possibility that repetition made participants focus on the photo itself as the source of relevant information for the judgment, while disregarding the content of the presented information. We addressed these issues in the second study.

\section{STUDY 2}

In the first study we showed that familiarity induced by subliminal exposure to a target's face reduced the impact of individuating information in judgments about the target. Our manipulation of repetition 
was subtle and the familiarity focused on the target. In the second study we attempted to induce familiarity not about the target but about the individuating information itself. We did this by making some participants familiar with some of the individuating information presented about the target. This was achieved by repeating the gist of some of the information in different parts of the experimental session. Given that both priming accounts and information integration accounts would predict that repeated information would have greater impact on judgments, our prediction that such repetition would reduce the impact of the repeated information was a strong test of the hypothesis.

In order to better claim that the reduction in impact of individuating information was the result of non-analytic processing, we also in this second experiment provided the target with category membership. If familiarity reduces analytic processing and thus the impact of individuated information on judgments, it is also expected to heighten the impact of categorical information on those judgments.

\section{Method}

\section{Participants and Design}

Participants were 55 female and 18 male ISPA undergraduates. They were randomly distributed to the eight cells of a 2 (no prior exposure vs. prior exposure to evidence) $\times 2$ (exculpatory vs. incriminating evidence) $\times 2$ (skinhead vs. priest category membership) between subjects factorial design.

\section{Procedure}

Participants completed the experiment in groups of 5-15, with random assignment to conditions within each session. As in Study 1 the experimenter informed participants that they were going to participate in a study of people's abilities to serve as a jury member considering a criminal case, and that they should read carefully all the instructions and information that were presented in a booklet.

The information presented in the booklet tried as realistically as possible first an interview with a detective inspector about the way criminal investigations typically proceed and then information about a specific case and its suspect.

Manipulation of repetition of information During the interview, after stating that it is necessary to get as much information as possible about the suspect, the detective gave some examples of the categories of information he looked for (activities, life history, alibi, etc.) in an investigation. In the prior exposure conditions, the inspector then went on to give examples that were closely similar in wording to the items of information later provided regarding the specific case. Thus, the inspector said (among other things): "For example, it is important to know if a suspect is seen leaving the scene of the crime some minutes before the crime took place" and "It is relevant to know whether or not someone was able to identify the assailant." These statements were phrased neutrally (e.g., saying "whether or not" someone could identify the aggressor), but were similar to a neutral item, two exculpatory, and two incriminating items of evidence provided later about the specific crime for all participants (e.g., one incriminating item stated that "someone was able to identify the aggressor"). In the no prior exposure condition the inspector gave examples that were unrelated to the evidence items included in the report on the specific suspect. (e.g., "it's important to find all the witnesses of the crime"). 
Presentation of category and individuating information Participants received the same information about the crime, suspect, and evidence described in Study 1 with two exceptions: no photo of the suspect was presented, and the brief description of the suspect included the information that he was either a skinhead or a priest.

Participants made the same four judgments of perceived guilt as in Study 1, and the same three auxiliary judgments of how sufficient the information provided was, how incriminating the evidence was, and how aggressive the suspect seemed. However, in this study judgments were made on 11-point scales. As part of a later general questionnaire about research participation, participants were asked to recall the occupation of the suspect. To assess participant motivation, we also asked how interesting they found participation in this study (responses were made on a 11-point scale anchored by 1 - not interesting at all to 11 - very interesting).

\section{Results and Discussion}

Data from three participants whose responses were outliers with regard to guilt judgments and also compromised the normal distribution and homogeneity assumptions of ANOVA were excluded from analyses. Some participants failed to answer all questions, resulting in different $N$ for some analyses.

\section{Checks on the Effectiveness Manipulations}

Corroborating pre-testing, the suspect was perceived as more violent when described as a skinhead $(M=7.04)$ than as a priest $(M=5.37), t(60)=3.90, p<.001, \eta^{2}=0.20$. The suspect was also seen as more violent when incriminating $(M=6.91)$ rather than exculpatory $(M=5.51)$ evidence was presented, $F(60)=3.26, p<.002, \eta^{2}=0.15$, but this effect did not qualify the category information effect (the interaction, $F<1$ ).

Participants recalled the occupation of the suspect equally well when he was a priest $(90 \%)$ and when he was a skinhead $(85 \% ; p<.569)$. None of the other variables affected correct identification of the category information.

It appeared that the criminal trial context and instructions had a positive impact on participants' motivation to process information in the study. Responses to the question of how interesting participants found the study revealed mean motivation to be well above the midpoint of the scale $(M=7.24, S D=1.85)$.

\section{Judgments of Guilt}

Responses to the four items used to assess guilt (Cronbach alpha $=.77$ ) were averaged, with high numbers reflecting higher guilt. We tested our hypotheses by performing planned contrasts within the overall design $($ Mse $=2.84)$.

First, we expected the impact of the individuating evidence to be moderated when the information was familiar compared to when it was not, indicating that familiarity reduced analytic processing and replicating the results of Study 1. The manipulation of information had the expected impact on judgments, $t(62)=3.07, p<.003$, with incriminating information leading to higher guilt judgments $(M=6.07)$ than exculpatory information $\left(M=4.81, \eta^{2}=0.13\right)$. We expected this effect to be qualified by prior exposure. To test this hypothesis we contrasted the differential effect of incriminating and 


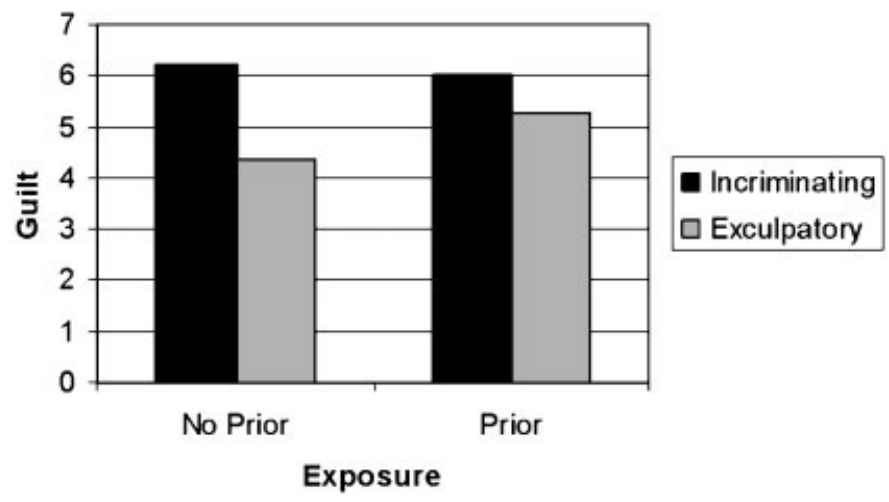

Figure 2. Guilt judgments as function of prior exposure and nature of individuating information, Study 2

exculpatory information in the prior exposure and no prior exposure conditions, $t(62)=1.48, p<.07$, $\eta^{2}=0.04$, one-tailed. Consistent with our expectations, post hoc comparisons showed that participants judged the defendant as guiltier in the incriminating evidence condition $(M=6.21)$ than in the exculpatory evidence condition, $(\mathrm{M}=4.35), t(62)=3.2, p<.002, \eta^{2}=0.14$, when there was no prior exposure to the information, but that this effect of evidence was not significant in the prior exposure condition $($ Minc $=5.93$, Mexc $=5.78 ; t(62)=1.13, p<.260)$ (Figure 2).

Second, we expected the impact of category membership information on judgments of guilt to be enhanced by previous exposure, since such an effect would reflect an increase in non-analytic processing. To test this hypothesis we contrasted the impact of category membership in the prior and no prior exposure conditions, $t(62)=1.94, p<.028, \eta^{2}=0.06$. As expected, post hoc comparisons revealed that in the no prior exposure condition, the skinhead $(M=4.99)$ and priest $(M=5.57)$ suspects were seen as equally guilty, $t<1$. In contrast, and consistent with our hypothesis, participants who had been previously exposed to some of the evidence judged the stereotypically aggressive skinhead to be marginally more guilty $(M=6.11)$ than the stereotypically less aggressive priest $(M=5.09)$, $t(62)=1.75, p<.08, \eta^{2}=0.04$ (Figure 3 ).

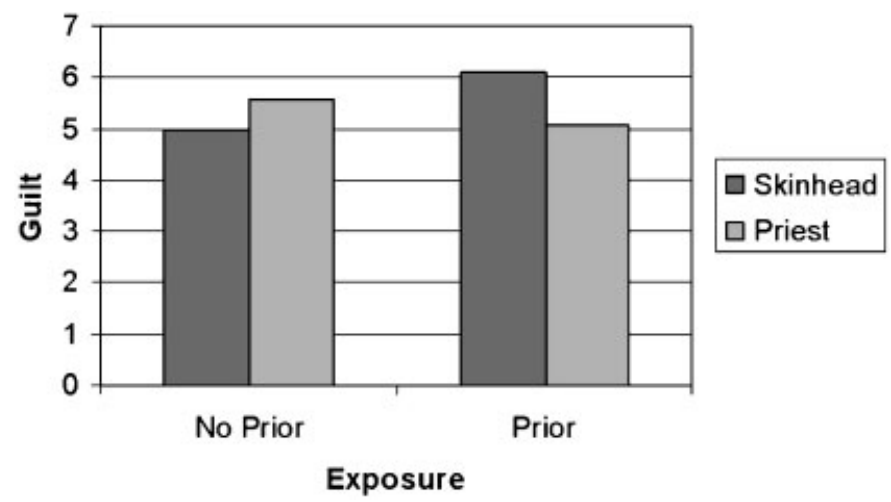

Figure 3. Guilt judgments as function of prior exposure and category label, Study 2 
This pattern of data suggested that inducing familiarity by previously exposing participants to some aspects of the evidence actually reduced the impact of the repeated information itself on judgments, as it at the same time tended to increase the impact of categorical processing on judgments.

Guided by the results of Study 1, we analyzed the impact of our manipulations on perceived sufficiency with planned contrasts using the overall error term from the full ANOVA design, Mse $=4.82$. As in Study 1, perceived sufficiency was influenced by the nature of information, $t(61)=3.06, p<.003$, with incriminating information leading to perceptions of greater sufficiency than exculpatory information $\left(M=5.22\right.$ vs. $\left.3.56, \eta^{2}=0.13\right)$. This effect was, as in Study 1 , qualified by an interaction with prior exposure, $\left(t(61)=2.77, p<.007, \eta^{2}=0.11\right.$, with evidence having a significant effect when there was no prior exposure $\left(\operatorname{Minc}=5.85, \operatorname{Mexc}=2.70 ; t(61)=4.15, p<.001, \eta^{2}=0.22\right)$, but not when there was prior exposure $(\mathrm{Minc}=4.58, \mathrm{Mexc}=4.42 ; t<1)$. Prior exposure did not by itself induce higher perceived sufficiency $(F<1)$, ruling out any role it might have in the exposure effect found here.

Also as in Study 1, however, perceptions of information sufficiency were related to increased judgments of guilt $(r=.46, t(68)=4.25, p<.001)$ in both exposure conditions (although more strongly when there was no prior exposure, $r=.53$, consistent with the finding that the individuating information had more impact when there was no prior exposure). Controlling for sufficiency $($ Mse $=2.52$ ) eliminated the main effect of the nature of the information, $t<1$. Since exposure did not impact sufficiency judgments, however, it is not surprising that its inclusion as a covariate did not eliminate the significance of the contrast reflecting the exposure $X$ category information interaction, $t(60)=2.27$, $p<.02$, actually making its impact even more evident $\left(\eta^{2}=0.08\right)$.

As expected, the preponderance of incriminating evidence was once again seen as more incriminating $(M=5.91)$ than the preponderance of exculpatory information $(M=3.86), t(61)=4.16$, $p<.001, \eta^{2}=0.25$, Mse $=4.01$. Unlike in Study 1 , this effect did not completely disappear in the prior exposure condition but instead became marginal $(\mathrm{Minc}=5.61, \mathrm{Mexc}=4.42 ; t(61)=1.91, p<.06$, $\left.\eta^{2}=0.05\right)$.

Adding perceptions of how incriminating the evidence was as a covariate, $F(1,61)=40.66$, $p<.0001$, in the guilt analysis eliminated (as expected) the impact of the nature of the evidence. It also however increased the significance of the contrast reflecting the exposure $\mathrm{X}$ category information interaction, $t(60)=2.33, p<.023, \eta^{2}=0.08$, perhaps reflecting that fact that in Study 2 non-analytic processing was revealed not only by reduced sensitivity to the individuating evidence, but also by increased reliance on stereotypic category-based information.

In sum, our central claim, that stimulus repetition reduces analytic processing of presented information, was replicated in this study even when familiarity was induced not about the target but about the individuated information itself. As our results make clear, even when it was the information itself that was repeated to induce familiarity, this rendered highly relevant individuating information to be less, rather than more, influential in judgments about a target person. This reduced sensitivity to individuating information was coupled with a nearly significant increase in reliance on category-based information. This pattern of results is consistent with the idea that prior exposure induced familiarity, which is associated with decreased analytic individuating processing and increased non-analytic categorical processing.

\section{Meta-analysis}

To examine the consistency of the findings that prior exposure reduced analytic individuating processing across studies, we computed the Stouffer Combined Test, with the relevant contrasts from 
both studies. The significance of this test, $Z c=3.75, p<.001$ indicated that there was highly reliable evidence across studies for a reduction in analytic processing following prior exposure.

\section{GENERAL DISCUSSION}

We hypothesized that the experience of familiarity intervenes in information processing by decreasing analytic processing. This led us to expect that in a person perception setting individuals familiar with the situation would rely less on individuating information whether forming an impression or making a judgment. In the two studies presented here, we promoted familiarity either by subliminally presenting a photo of the target (Study 1) or repeating the relevant individuating information about the target (Study 2). Consistent with our claim that repetition triggers reduced analytic processing, participants' judgments in both studies were less sensitive to the quality of the presented information, regardless of how familiarity was induced. Corroborating the idea that familiarity reduces analytic processing, the second study showed in addition that participants' judgments tended to also be more sensitive to category information whenever they experienced the situation as familiar.

Our experiments were able to eliminate as a cause of these results two effects already well established in the literature: mere exposure and social judgeability. Prior exposure is known to increase preference and liking, but this effect was not seen in either study. No main effect was associated with repetition. All the relevant effects were interactions. That is, although prior exposure decreased perceived guilt in the presence of incriminatory information (perhaps reflecting benevolent, positive, or liking judgments), it increased perceived guilt in the presence of exculpatory information in both studies. Under certain conditions individuals have also been shown to feel more entitled or qualified to make quick judgments following prior exposure to information about a topic, the social judgeability effect. However, neither study provided any evidence that participants familiar with the situation felt they had a more sufficient basis for their judgments, nor was judgeability a mediator of familiarity's effect on processing.

The majority of studies within the person perception field have focused on the impact of category activation and use in different social judgments and how this strategy economizes aspects of information processing, such as item encoding, resource allocation, and response generation (see Bodenhausen \& Lichtenstein, 1987; Bodenhausen \& Wyer, 1985; Macrae, Bodenhausen, Schloerscheidt, \& Milne, 1999; Macrae, Milne, \& Bodenhausen, 1994). One set of studies (Pendry \& Macrae, 1994) has particularly focused on the roles that motivation and capacity play in perceivers' tendency to think about others in either an individuated or category-based manner. Our results, together with those of Smith et al. (2006), show that although motivation and capacity are two highly relevant moderators of how information about a target person is processed, they do not fully explain the regulation of such dual processing. Our studies call attention to the importance of a "match" between received information and stored structures, as hypothesized by Fiske and Neuburg (1990), for processing mode. Our findings go further, however, by showing that this "match" need not be limited to just a match of category information. In addition, our second study offers further support for the idea of a particular dynamic between analytic and non-analytic processing, as dual processing models of person perception hypothesize. Although both processes co-occur, judgments tend to be inversely impacted by category and individuated information, with an increase in the influence of one being associated with a decrease in the influence of the other.

The fact that such matches may induce familiarity which in turn reduces analytic processing might help explain the impact that feature typicality has on categorization and stereotyping. For example, exposure to typical and therefore familiar Afrocentric facial features has been found to activate stereotypic inferences (Blair, Judd, \& Fallman, 2004; Livingston \& Brewer, 2002; Maddox \& Gray, 
2002). From our perspective, these effects appear to constitute another example of the typicality of those features activating familiarity, and thus decreased reliance on bottom-up processing coupled with increased reliance on category-based processing.

Several aspects of this research warrant further investigation. First, the primary hypothesis tested in these studies was derived from our claim (Garcia-Marques \& Mackie, 2000, 2001) that familiarity is a diffuse positively valenced feeling that intervenes in information processing by signaling that non-analytic processing is viable or appropriate. Although the results of these studies are consistent with this view, we did not provide direct evidence that it is the feeling of familiarity that mediates the obtained processing effects. Consistent with this view, Smith et al. (2006) showed that drawing explicit attention to the possibility of multiple exposure to information eliminated its effect on processing, just as similar manipulations reduce the "false fame" effect (Bacon, 1979). Nevertheless, direct evidence of both the effect of prior exposure on feelings of familiarity, and of the mediational role of these feelings in triggering different processing modes is necessary to appropriately substantiate our claim.

Second, our claim that familiarity triggers non-analytic processing implies that other subjective states that "feel like" familiarity should do so also. We have argued, for example (Garcia-Marques et al., 2004), that the experience of familiarity is inherently positive. This suggests in turn that positive affective states, such as good moods, might well trigger non-analytic processing (as they are known to do, Bless, Bohner, Schwarz, \& Strack, 1990; Mackie \& Worth, 1989) because they imply or are associated with, at least under certain conditions, familiarity (Garcia-Marques, 1999). To date no direct evidence speaks to the issue of whether the reduction in analytic processing following familiarity and the reduction in analytic processing in positive mood depend on the same mechanisms.

Third, if our claims are right, our manipulations of familiarity have an influence on behavior because such artificially induced familiarity interferes with the "natural" role of familiarity in information processing. However, every instance of person perception involves multiple occurrences of the activation of familiarity associated with expectancies, which can then interact with the nature of any information given. For example, activation of the priest category label might make information about the kind of crime like the one we used more surprising than activation of the skinhead label. Equally, exculpatory information is perhaps less surprising when a priest is the suspect than when a skinhead is a suspect (Skowronski, 2002). Thus, the experimental induction of familiarity might privilege categorical information (as it reduces analytic and increases non-analytic processing), but the very activation of that information might activate several other processes that undermine the feeling of familiarity. Note that the results of our second study, involving category activation, were not as strong as the results of the first study, suggesting that this might be the case. Fully understanding these effects requires a more systematic study of how violations of expectancies undermine experimentally manipulated familiarity.

A fourth set of questions has to do with when targets will be perceived as familiar. Some research findings suggest that the nature of a prior encoding experience is a critical determinant of person categorization (Blair, 2002). Thus, prior categorization (the way a target is originally perceived) may determine whether familiarity is activated in any specific later "retrieval" (not necessary conscious) situation, such that "congruent categorizations" will register as familiar but different categorizations will not. Thus, the processing goals active during the first encounter with a target and during subsequent exposure may moderate the impact of "repetition" on processing that occurs during that subsequent exposure.

In addition to this obvious boundary condition, other variables are also expected to moderate this effect. Our claim is that processors are sensitive to familiarity and that familiarity triggers non-analytic processing. As a consequence, any disruption of such familiarity (the presence of incongruent or unexpected information, for example) will affect this mode of processing, as repeatedly demonstrated in the literature (see Johnston \& Hawley, 1994; Stangor \& McMillan, 1992). In addition, the level of perceived familiarity may itself be influenced by the level of processing a stimulus receives. Thus, a 
stimulus that at "first glance" might match a memory representation might well be seen as novel after receiving more attention. In fact, sustained attention to distinguishing, differentiating, or individuating details is likely to disrupt any experience of familiarity. Thus, any factor able to motivate individuals to attend to details is expected to influence the experience of familiarity. Partial support for this assumption was obtained by Claypool et al. (2004), who showed that issue relevance disrupted the impact of familiarity on the mode with which persuasive material was processed.

One of the implications of our findings is that multiple contextual factors can induce familiarity, which will in turn induce less analytic processing of relevant information. Thus, familiarity can arise from such different sources as a familiar room, a familiar group of people, or a familiar message frame, and all of these might reduce processing. Consider the practical implication of such effects: Having the same professor deliver a lecture in the same monotone voice in the same room with the same members of the class present — particularly if they all sit in the same seats - might not be the best way to induce analytic processing of lecture content. Such considerations also point to the value of manipulations that undermine or lead to the misattribution of activated feelings of familiarity.

Our findings also have practical implications for the legal settings that we modeled in our experiment. When freedom versus incarceration, or even life versus death, might depend on information processing, the intrusion of factors that reduce careful processing, especially if they also increase stereotypic bias, are particularly dangerous. Attorneys sometimes strategically attempt to activate sympathy for a defendant by making juries more "familiar" with the suspect. Although activation of mere exposure mechanisms might justify this strategy, it is worth noting that any activated feelings of familiarity can also reduce careful processing of relevant information and increase reliance on stereotypic information (of course, if a preponderance of evidence is incriminating, or the defendant is a member of a highly valued group, this is not a bad thing). Our findings are also relevant to the ongoing debate about the impact of pre-trial publicity on trial outcomes (e.g., Greene \& Wade, 1988; Honess \& Charman, 2002). Research on this topic has been couched largely in terms of whether exposure to additional, legally irrelevant, and often inflammatory details about the crime or the accused bias the content of jury decision making (Carrol et al., 1986; Steblay, Besiveric, Fulero, \& JimenezLorente, 1999). Our data suggest a more thorough going and generalized effect: when either the crime or the alleged criminal seems familiar, careful processing of case-specific information can be reduced while reliance on category-based, stereotypic and heuristic judgments can be increased. That vague glimmer of recognition induced by having seen the accused in the morning paper or on the morning news can have serious and significant information processing consequences.

\section{ACKNOWLEDGEMENTS}

This research was supported by a Fundação para a Ciência e Tecnologia grant POCTI-PSI \#42239/01 to Teresa Garcia-Marques and by National Science Foundation Grant \#BCS-9975204 to Diane Mackie. We are grateful to Alexandre Fernandes for assistance with data collection and to Pedro Brazão who worked on the pre-test of material used in these studies.

\section{REFERENCES}

Abelson, R. P. (1994). A personal perspective in social cognition. In P. Devine, D. L. Hamilton, \& T. M. Ostrom (Eds.), Social cognition: Impact on social psychology (pp. 15-37). New York: Academic Press. 
Anderson, N. H. (1965). Averaging versus adding as a stimulus combination rule in impression formation. Journal of Experimental Psychology, 70, 394-400.

Bacon, F. T. (1979). Credibility of repeated statements: Memory for trivia. Journal of Experimental Psychology: Human Learning and Memory, 5, 241-252.

Blair, I. V. (2002). The malleability of automatic stereotypes and prejudice. Personality and Social Psychology Review, 6, 242-261.

Blair, I. V., Judd, C. M., \& Fallman, J. L. (2004). The automaticity of race and Afrocentric facial features in social judgments. Journal of Personality and Social Psychology, 87, 763-778.

Bless, H., Bohner, G., Schwarz, N., \& Strack, F. (1990). Mood and persuasion: A cognitive response analysis. Personality and Social Psychology Bulletin, 16, 331-345.

Bodenhausen, G. V., \& Lichtenstein, M. (1987). Social stereotypes and information-processing strategies: The impact of task complexity. Journal of Personality and Social Psychology, 52, 871-880.

Bodenhausen, G. V., \& Wyer, R. S., Jr. (1985). Effects of stereotypes on decision making and informationprocessing strategies. Journal of Personality and Social Psychology, 48, 267-282.

Bodenhausen, G. V. (1990). Stereotypes as judgmental heuristics: Evidence of circadian variations in discrimination. Psychological Science, 1, 319-322.

Brewer, M. B. (1988). A dual process model of impression formation. In R. S. Wyer, \& T. K. Srull (Eds.), Advances in social cognition (Vol. 1, pp. 1-44). Mahwah, NJ: Erlbaum.

Bruce, V., \& Young, A. (1986). Understanding face recognition. British Journal of Psychology, 77, 305327.

Carrol, J. S., Kerr, N. L., Alfini, J. J., Weaver, F. M., MacCount, R. J., \& Feldman, V. (1986). Free press and fair trial: The role of behavioural research. Law and Human behaviour, 10, 187-202.

Claypool, H. M., Mackie, D. M., Garcia-Marques, T., McIntosh, A., \& Udall, A. (2004). The effects of personal relevance and repetition on persuasive processing, Social Cognition, 22, 310-335.

Dreben, E. K., Fiske, S. T., \& Hastie, R. (1979). The independence of evaluative and item information: Impression and recall order effects in behavior-based impression formation. Journal of Personality and Social Psychology, 37, 1758-1768

Eich, J. M. (1982). A composite holographic associative recall model. Psychological Review, 89, 627-661.

Fiske, S. (1982). Schema triggered affect: Applications to social perception. M. S. Clark, \& S. T. Fiske (Eds.), Affect and cognition: The seventeenth annual carnegie symposium on cognition (pp. 55-78). Hillsday, NJ: Lawrence Erlbaum Associates Inc.

Fiske, S. R., \& Pavelchak, M. A. (1986). Category-based versus piecemeal-based affective responses: Developments in schema-triggered affect. In R. M. Sorrentino, \& E. T. Higgins (Eds.), Handbook of motivation and cognition: Foundations of social behavior (pp. 167-203). New York, NY: Guilford press.

Fiske, S. T. (1980). Attention and weight in person perception: The impact of negative and extreme behavior. Journal of Personality and Social Psychology, 38, 889-906.

Fiske, S. T., Neuberg, S. L., Beattie, A. E., \& Milberg, S. J. (1987). Category-based and attribute-based reactions to others: Some informational conditions of stereotyping and individuating processes. Journal of Experimental Social Psychology, 23, 399-427.

Fiske, S., \& Neuburg, S. L. (1990). A continuum of impression formation form category-based to individuating processes: Influences of information and motivation on attention and interpretation. In L. Berkowitz (Ed.), Advances in experimental social psychology (Vol. 23, pp. 1-74). San Diego: Academic Press.

Garcia-Marques, T. (1999). The mind needs the heart. The mood-as-regulation mechanism hypothesis. Dissertação de Doutoramento apresentada na Universidade de Lisboa.

Garcia-Marques, T., Mackie, D. M., Claypool, H. M., \& Garcia-Marques, L. (2004). Positivity can cue familiarity. Personality and Social Psychology Bulletin, 30, 1-9.

Garcia-Marques, T., \& Mackie, D. M. (2000). The positive feeling of familiarity: Mood as an information processing regulation mechanism. In J. Forgas, \& H. Bless (Eds.), The message within: The role of subjective experiences in social cognition and behavior. Philadelphia: Psychological Press.

Garcia-Marques, T., \& Mackie, D. M. (2001). The feeling of familiarity as a regulator of persuasive processing. Social Cognition, 19, 9-34.

Gillund, G., \& Shiffrin, R. M. (1984). A retrieval model for both recognition and recall. Psychological Review, 91, 1-67.

Greene, E., \& Wade, R. (1988). Of private talk and public print: General pre-trial publicity and juror decisionmaking. Applied Cognitive Psychology, 2, 123-135

Hintzman, D. L. (1988). Judgments of frequency and recognition memory in a multiple-trace memory model. Psychological Review, 95, 528-551. 
Honess, T. M., \& Charman, E. A. (2002). Members of the jury-Guilty of incompetence? Psychologist, 15, 72-75.

Humphreys, M. S., Bain, J. D., \& Pike, R. (1989). Differential ways to cue a coherent memory system: A theory for episodic, semantic and procedural tasks. Psychological Review, 96, 208-223.

Jacoby, L. L., \& Dallas, M. (1981). On the relation between autobiographical memory and perceptual learning. Journal of Experimental Psychology: General, 3, 306-340.

Johnston, W. A., \& Hawley, K. J. (1994). Perceptual inhibition of expected inputs: The key that opens closed minds. Psychonomic Bulletin and Review, 1, 56-72.

Judd, C. M., Park, B., Yzerbyt, V. Y., Gordijn, E., \& Muller, D. (2005). They show more intergroup bias and have stronger stereotypes than do I: Evidence from ethnic, gender, and nationality intergroup contexts. European Journal of Social Psychology, 35, 677-704.

Livingston, R. W., \& Brewer, M. B. (2002). What are we really priming? Cue-based versus category-based processing of facial stimuli. Journal of Personality and Social Psychology, 82, 5-18.

Mackie, D., \& Worth, L. T. (1989). Processing deficits and the mediation of positive affect in persuasion. Journal of Personality and Social Psychology, 57, 27-40.

Macrae, C. N., Bodenhausen, G. V., Schloerscheidt, A. M., \& Milne, A. B. (1999). Tales of the unexpected: Executive function and person perception. Journal of Personality \& Social Psychology, 76, 200-213.

Macrae, C. N., Milne, A. B., \& Bodenhausen, G. V. (1994). Stereotypes as energy-saving devices: A peek inside the cognitive toolbox. Journal of Personality \& Social Psychology, 66, 37-47.

Maddox, K.B., \& Gray, S. (2002). Cognitive representations of African Americans: Re-exploring the role of skin tone. Personality and Social Psychological Bulletin, 28, 250-259.

Murdock, B. B., Jr. (1982). A theory for the storage of and retrieval of item and associative information. Psychological Review, 89, 609-662.

Neuberg, S. L., \& Fiske, S. T. (1987). Motivational influences on impression formation: Outcome dependency, accuracy-driven attention, and individuating processes. Journal of Personality \& Social Psychology, 53, 431444.

Pendry, L. F., \& Macrae, C. N. (1994). Stereotypes in mental life: The case of the motivated but thwarted tactician. Journal of Experimental Social Psychology, 30, 303-325.

Reder, L. M., \& Ritter, F. E. (1992). What determines initial feelings of knowing? Familiarity with question terms not with the answer. Journal of Experimental Social Psychology: Learning, Memory and Cognition, 18, 435451.

Schunn, C. D., Reder, L. M., Nhouyvanisvong, A., Richards, D. R., \& Stroffolino, P. J. (1997). To calculate or not to calculate: a source activation confusion model of problem familiarity's role in strategy selection. Journal of Experimental Psychology: Learning, Memory, and Cognition, 23, 3-29.

Skowronski, J. J. (2002). Honesty and intelligence judgments of individuals and groups: The effects of entityrelated behavior diagnosticity and implicit theories. Social Cognition, 20, 136-169.

Sloman, S. A. (1996). The empirical case for two systems of reasoning. Psychological Bulletin, 119, 3-22.

Smith, E. R., Miller, D. A., Maitner, A. T., Crump, S. A., Garcia-Marques, T., \& Mackie, D. M. (2006). Familiarity can increase stereotyping. Journal of Experimental Social Psychology, 42, 471-478.

Smith, E. R., \& DeCoster, J. (2000). Dual process models in social and cognitive psychology: Conceptual integration and links to underlying memory systems. Personality and Social Psychology Review, 4, 108-131.

Stangor, C., \& McMillan, D. (1992). Memory for expectancy-congruent and expectancy-incongruent information: A review of the social and developmental literatures. Psychological Bulletin, 111, 42-61.

Steblay, N. M., Besiveric, J., Fulero, S. M., \& Jimenez-Lorente, B. (1999). The effects of pretrial publicity on juror verdicts: a meta-analytic review. Law and Human Behavior, 23, 219-235.

Yzerbyt, V. Y., Dardenne, B., \& Leyens, J. P. (1998). Social judgeability concerns in impression formation. In V. Y. Yzerbyt \& G. Lories (Eds.), Metacognition: Cognitive and social dimensions (pp. 126-156). Thousand Oaks, CA: Sage. 